\title{
A Novel Organic Rankine Cycle System with Improved Thermal Stability and Low Global Warming Fluids
}

\author{
Angad S Panesara , Robert E Morgan, Nicolas DD Miché and Morgan R Heikal \\ Centre for Automotive Engineering, University of Brighton, BN2 4GJ, United Kingdom
}

\begin{abstract}
This paper proposes a novel Organic Rankine Cycle (ORC) system for long haul truck application. Rather than typical tail pipe heat recovery configurations, the proposed setup exploits the gaseous streams that are already a load on the engine cooling module. The system uses dual loops connected only by the Exhaust Gas Recirculation (EGR) stream. A water blend study is conducted to identify suitable mixtures for the High Temperature (HT) loop, while the Low Temperature (LT) loop utilises a Low Global Warming (GWP) Hydrofluoroether.
\end{abstract}

\section{Introduction}

The use of ORC on long haul Heavy Duty Diesel Engines (HDDE) is a possible way to improve the specific fuel consumption. The two widely proposed organic working fluids for HDDE include R245fa and ethanol [1]. Also, the use of Rankin Cycle (RC) with water has been suggested [1]. The proposed fluids are not without their specific challenges. R245fa and ethanol's stable temperature limits are over $100^{\circ} \mathrm{C}$ below the typical $\left(400-500^{\circ} \mathrm{C}\right)$ EGR temperatures $[2,3]$. When these two fluids are compared, R245fa is non-flammable but has a high GWP (950 yrs.). Ethanol on the other hand is highly flammable and has a low auto-ignition temperature of $363^{\circ} \mathrm{C}$. Unfortunately, a $\mathrm{RC}$ solution also has its drawbacks, since water as a working fluid is only superior over organic fluids above $500^{\circ} \mathrm{C}$ [1]. Water is also the wettest fluid, requiring large amounts of superheat to avoid turbine damage. The additional drawback of using water is its high freezing temperature of $0^{\circ} \mathrm{C}$.

\section{Overview of the Blend study}

In the absence of current pure fluids meeting the complex requirements of HDDE application, fluid blends may present an alternative avenue. As the resurgence of ORC systems for HDDE is relatively new, the present research on blends is inadequate to ascertain their suitability for vehicle application. This paper explores the use of binary water blends to provide desired properties and characteristics which can be tailored by varying the mass fractions. To screen the list of documented water blends [4], the developed criteria shown in Table 1 were used. Three positive homogeneous azeotropes were selected. Namely, water-diacetone alcohol, water-2butanol and water-acetonitrile with water mass fraction of $0.77,0.26$ and 0.15 respectively, forming an azeotropic composition at 1 bar. Also, three

\footnotetext{
${ }^{\text {a }}$ Corresponding author : A.Panesar@brighton.ac.uk
}

This is an Open Access article distributed under the terms of the Creative Commons Attribution License 2.0, which permits unrestricted use, distribution, and reproduction in any medium, provided the original work is properly cited. 
zeotropic blends of water are considered. Namely, water-ethanol, water-methanol and water-acetone with water mass fraction of 0.25 and 0.75 in all 3 blend cases.

The temperature-pressure relationship of zeotropic fluids differs from that of pure and azeotropic fluids during vaporization i.e. the composition of the vapour and the liquid phase of the blend at the vapor-liquid equilibrium state are never the same. The dissimilar volatilities of the components cause the mixture boiling to span a range between the bubble point and dew point temperature. On the other hand, azeotropic blends are those in which both dew point and bubble point curves are touching each other at least at a single point indicating the same composition in the vapour and liquid phase. During vaporisation an azeotropic blend behaves as a pure fluid, the composition of the vapour and the liquid remain essentially unchanged.

Table 1. Screening criteria used to identify the second constituent of water blend.

\section{Molecular screening}

- Structure excludes Chlorine and Bromine molecules

- Number of atoms in a molecule $\leq 20$

\section{Thermo-physical screening}

- T,freezing $\leq-40^{\circ} \mathrm{C}$

- P,condensing for blend $@ 100^{\circ} \mathrm{C} \geq 1 \mathrm{bar}$

- For azeotropes : only homogeneous, positive, with water content $15-80 \%$ by mass

\section{Environmental and safety screening}

- Ozone Depletion Potential $=0$

- Global Warming Potential $\leq 150$ yrs.

- Health (NFPA) $\leq$ Moderate (2)

- Flammability (NFPA) $\leq$ Serious (3)

- Instability $(\mathrm{NFPA}) \leq \operatorname{Minimal}(0)$

- $\mathrm{T}$, auto-ignition $>360^{\circ} \mathrm{C}$

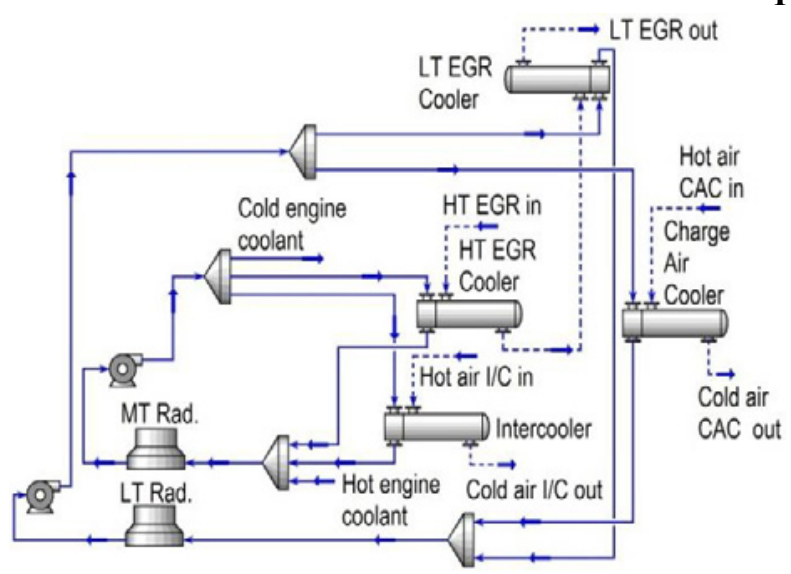

Table 2. Quantities and Qualities of the heat sources.

\begin{tabular}{|c|c|c|c|c|c|c|}
\hline & $\begin{array}{l}\text { C100 } \\
\text { Duty } \\
{[\mathrm{kW}]}\end{array}$ & $\begin{array}{l}\text { Inlet } \\
{\left[{ }^{\circ} \mathrm{C}\right]}\end{array}$ & $\begin{array}{l}\text { Dutlet } \\
{\left[{ }^{\circ} \mathrm{C}\right]}\end{array}$ & $\begin{array}{l}\text { B50 } \\
\text { Duty } \\
{[\mathrm{kW}]}\end{array}$ & & $\begin{array}{l}\text { Dutlet } \\
{\left[{ }^{\circ} \mathrm{C}\right]}\end{array}$ \\
\hline HT EGR & 68.9 & 466 & 200 & 36.1 & 388 & 152 \\
\hline $\mathrm{I} / \mathrm{C}$ & 10.3 & 145 & 121 & 0 & & \\
\hline Engine cooling & 52.5 & 97 & 90 & 38.1 & 97 & 90 \\
\hline LT EGR & 26.1 & 200 & 95 & 8.5 & 152 & 95 \\
\hline HT CAC & 49.5 & 200 & 85 & 12.8 & 152 & 85 \\
\hline LT CAC & 9.8 & 85 & 62 & 6.7 & 85 & 50 \\
\hline LT Rad. & 75.6 & 77 & 65 & 21.3 & 79 & 65 \\
\hline HT Rad. & 131.7 & 97 & 90 & 74.2 & 97 & 90 \\
\hline Air LT Rad. & & 20 & 35 & & 20 & 35 \\
\hline Air HT Rad. & & 35 & 50 & & 35 & 50 \\
\hline Exuaust & 99.3 & 302 & 95 & 42.2 & 290 & 95 \\
\hline
\end{tabular}

Figure 1. Cooling module of the HDDE.

The selected HDDE model for ORC is a 10 litre, 6 cylinder, 2 stage turbo-charged engine using EGR only (35-45\%) and high injection pressures (2300-2500 bar) to meet the Euro 6 oxides of nitrogen. The layout of the engine cooling module is depicted in Fig. 1. Table 2 shows the qualities and quantities in the streams at a cruise (B50) and a high load (C100) condition.

\section{Results and Discussion on blends}

Fig. 2 shows the net power for the subcritical dry saturated vapour expansion ORC at C100 and B50 using the 9 compositions for a widely proposed parallel EGR and Exhaust (EXH) heat recovery arrangement. Boundary conditions and assumptions used in the calculations are given in Table 3. At $\mathrm{C} 100$, the highest power is achieved using water-acetonitrile $(19.5 \mathrm{~kW})$. The 3 compositions, water(0.25)-ethanol, water(0.25)-methanol, and water-2butanol give results similar to each other $(17.3-17.9 \mathrm{~kW})$. Followed by water(0.75)-ethanol, water(0.75)-methanol (16.6-16.8kW). Also, water- 
diacetone alcohol and water(0.25)-acetone give nearly equal power (15.8-15.9kW). The worst performance $(14.9 \mathrm{~kW})$ is obtained using water(0.75)-acetone. The results at B50 also show similar trend in superior performance by water-acetonitrile, water(0.25)-ethanol, water(0.25)-methanol and water-2butanol.

An important limitation of pure and azeotropic fluids is the isothermal boiling, leading to large levels of heat exchanger irreversibility's. However, the temperature glide (which is relative to both the composition and pressure) exhibited by the zeotropic fluids results in a variation in temperature during isobaric phase change. This produces a better temperature match to a finite thermal source, increasing average heat addition temperature and reducing heat exchanger irreversibility's. Furthermore in the condenser, due to having an increased average heat rejection temperature, the blend has a larger heattransfer driving force to achieve the same condensation exit temperature. Unfortunately, as reduction in the condensing temperature has more influence on thermal efficiency than increase in evaporation temperature, zeotropic fluids exhibit lower thermal efficiency for similar evaporator inlet and condenser exit temperatures.

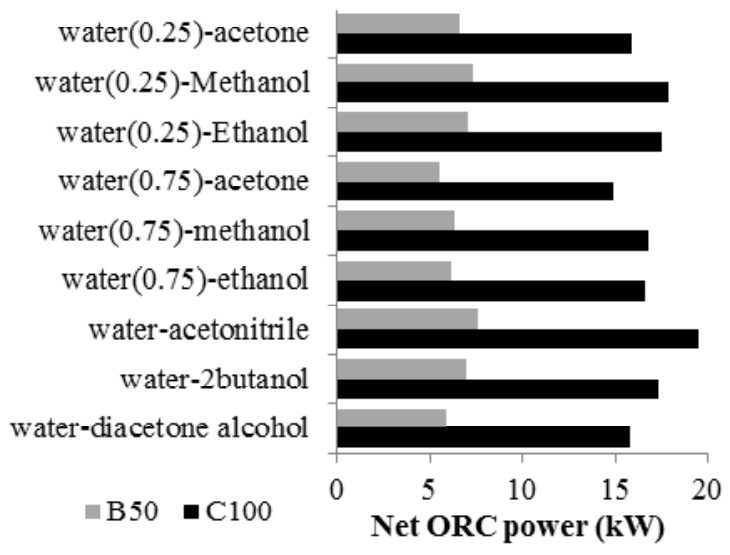

Table 3. Simulation boundary and assumptions.

Figure 2. Performance at the selected points.

Water when mixed with low freezing point fluids [5] will improve frost protection. With freezing points for pure 2 butanol, ethanol, methanol and acetone ranging between -94.7 and $-114.7^{\circ} \mathrm{C}$, when mixed with only $25 \%$ water by mass $(26 \%$ in case of 2 butanol) the freezing point of all the blends will be lower than $-50^{\circ} \mathrm{C}$. Blends of ethanol, methanol and acetone with a much higher water content of $75 \%$ will increase this value to around -15 to $-25^{\circ} \mathrm{C}$. The least frost protection $\left(-8\right.$ to $\left.-10^{\circ} \mathrm{C}\right)$ will be shown by the water-diacetone alcohol blend, due to a much lower freezing point $\left(-44^{\circ} \mathrm{C}\right)$ of diacetone alcohol and large $(77 \%)$ water mass fraction. Acetonitrile also has similar freezing point as that of diacetone alcohol but due to lower $(15 \%)$ water mass fraction the blends freezing point will be around -25 to $-30^{\circ} \mathrm{C}$.

Water is non-flammable and thermally stable at any practical operating temperatures. Binary blends with water as a component will have improved thermal stability as water will repress thermal decomposition. Pure diacetone alcohol shows the least National Fire Protection Association (NFPA) [5] flammability rating ( 2 i.e. moderate hazard) compared to all the other pure fluids ( 3 i.e. serious hazard). The $77 \%$ water mass fraction with diacetone alcohol may result in a non-flammable blend with the highest decomposition temperature. Water-diacetone alcohols results will be closely followed by ethanol, methanol and acetone blends with $75 \%$ water by mass fraction. 2butanol, ethanol, methanol and acetone with only $25 \%$ water by mass ( $26 \%$ in case of 2 butanol) will show much lower but nonetheless reduced flammability and increased decomposition temperatures.

Despite the highest net power, negligible change in flammability and decomposition will be shown by acetonitrile with only $15 \%$ water mass fraction. Water-diacetone alcohol offers the best flammability and decomposition security but the poor frost protection with a net power around $20 \%$ 
lower than water-acetonitrile, excludes it as a suitable blend candidate. Also, water-acetone blends are omitted as water-alcohol blends perform better with similar flammability and decomposition trends.

The concern with zeotropic blends significant glide in practical applications is that the working fluid fractionates in the tubes. In the evaporator, the lowest boiling point fluid will boil first and float to the top entering the expander first, leading to the accumulation of the higher boiling point fluid. This brings challenges such as incomplete phase change (wetness at expander inlet) and decreased heat-transfer performance. This has to be avoided by using superheated expansion rather than dry saturated vapour expansion. The higher amounts of superheat in the evaporators will ensure all the constituents are evaporated and the relative mass ratios are maintained. A similar phenomenon is also seen in the condenser and would require sufficient sub-cooling prior to pump inlet. A further observation is that the temperature glide exhibited by water-ethanol, water-methanol with water mass fractions of 0.25 and 0.75 in both the blend cases has not noticeably improved the net power over the azeotrope water-2butanol (Fig. 2).

All azeotropic blends are also not without similar challenges. In the case of water-acetonitrile the composition of azeotrope is affected by temperature/pressure i.e. the composition at which the azeotrope occurs changes. For a fixed composition, azeotrope composition shifts due to pressure swing and temperature glide is observed. Hence, this blend behaves as azeotrope in the condenser and zeotrope in the evaporator. This along with negligible change in flammability and decomposition precludes water-acetonitrile blend as a recommendation irrespective of the higher net power.

2 butanol (drying fluid) when mixed with $26 \%$ water (wetting fluid) by mass retains the dry expansion characteristics for the assumed expander efficiency and also avoids the use of a recuperator, simplifying packaging and controls. For heat recovery at the C100 case, the de-superheating load on the condenser is only $\sim 5 \%$ of the total condensing load. Furthermore, the water-2butanol blend forms a favourable positive homogeneous azeotrope. Firstly, for the temperature/pressure limits considered in this study, it remains as an azeotrope in the condenser and evaporator. Secondly, this blend may also be suited to slightly lower condensing temperatures of $\sim 90^{\circ} \mathrm{C}$ as seen in the Medium Temperature (MT) radiator. This is because water boils at $100^{\circ} \mathrm{C}, 2$ butanol boils at $99.5^{\circ} \mathrm{C}$, but the blend boils at a lower $88.5^{\circ} \mathrm{C}$. With ORC fitted onto a long haul truck experiencing uphill/downhill terrain, azeotrope water-2butanol along with other zeotropes which are less susceptible to fractionation $\left(\right.$ glide $<1^{\circ} \mathrm{C}$ ) and have higher water content may be optimal solutions.

\section{Novel ORC system}

The drawback of the EGR and EXH parallel heat recovery considered above is the incomplete utilisation of the thermal loads on the engine cooling module, mainly the incomplete utilisation of the LT EGR heat and no utilisation of the Charge Air Cooler (CAC) heat. Consider the case at B50, where depending on the ratio of latent heat to sensible heat of different blends the EGR is cooled from $388^{\circ} \mathrm{C}$ to $147-194^{\circ} \mathrm{C}$. The further cooling of the EGR down to $95^{\circ} \mathrm{C}$ is done by the LT radiator. With an addition of a flash expander over the EGR and EXH parallel heat recovery, the ORC system proposed in Fig. 3 utilises the LT EGR and CAC heat. The HT loop is the same EGR and EXH parallel heat recovery setup using water-2butanol as the working fluid that undergoes dry saturated vapour expansion and condenses in the HT condenser $\left(105^{\circ} \mathrm{C}\right)$. The LT loop consisting of a parallel heat recovery from the LT EGR and the CAC uses a trilateral flash expansion cycle with condensation in the LT condenser $\left(65^{\circ} \mathrm{C}\right)$.

The novelty of the cycle is in the:

- Higher utilisation of the present thermal loads (i.e. LT EGR and CAC) on the cooling module

- Only HT heat recovery from EXH, reducing the negative impact on the cooling module

- Use of azeotrope (water-2butanol) with $26 \%$ water content in the HT loop (improving thermal stability and reducing flammability)

- Use of a low GWP (110 yrs.) Difluoromethyl-Methyl-Ether (E152a) in the LT loop

- The use of trilateral flash cycle for higher overall conversion efficiency at low temperatures 


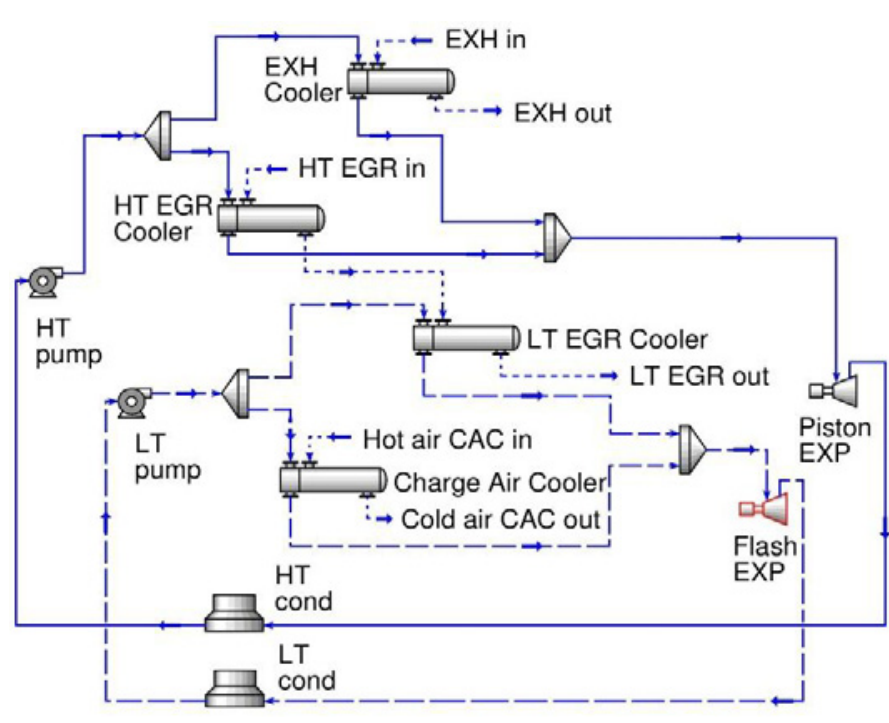

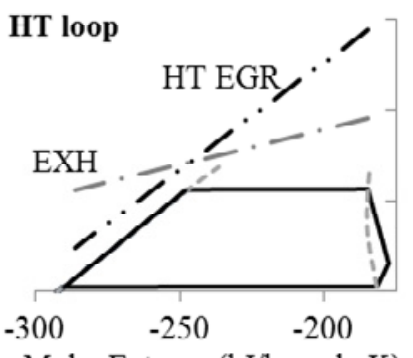

Molar Entropy (kJ/kgmole-K)

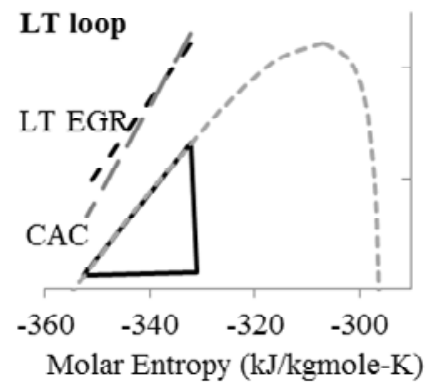

400

Figure 3. Proposed Novel ORC arrangement (with T-S diagram for HT and LT loop at B50).

When water-2butanol was used as a working fluid in EGR and EXH parallel heat recovery (Fig. 2) $7 \mathrm{~kW}(4.6 \%$ of engine shaft power) and $17.3 \mathrm{~kW}(5.8 \%$ of engine shaft power) was produced at B50 and $\mathrm{C} 100$. When the proposed ORC system (Fig. 3) is employed (without increase in the cooling load) the combined power of the two loops is $8.8 \mathrm{~kW}(5.8 \%$ of engine shaft power) and $21.9 \mathrm{~kW}(7.4 \%$ of engine shaft power) at B50 and C100. Furthermore the use of E152a ensures a compact flash expander (pressure ratio $\sim 2.7: 1$, volume flow ratio $\sim 14: 1$ ). Similar volume flow ratios are also seen in the 15:1 pressure ratio piston expander used in the HT loop.

\section{Conclusion}

The water blend study conducted to identify suitable mixtures for HT heat recovery in HDDEs has identified a positive homogenous azeotrope water-2butanol as a suitable candidate than the alternatives considered. With water mass fraction of $26 \%$ the thermal stability will improve and the flammability will reduce over pure 2 butanol. However, working fluid stopped-flow situations still have to be avoided. The use of this blend in the proposed novel dual loop ORC system with a low GWP fluid could increase the engine brake power by 5.8 to $7.4 \%$.

\section{References}

1. T. Wang et al., A review of researches on thermal exhaust heat recovery with Rankine cycle. Renewable and Sustainable Energy Reviews, 15(6): p. 2862-2871, (2011).

2. G. J. Zyhowski, Honeywell Refrigerants Improving the Uptake of Heat Recovery Technologies. www.honeywell-orc.com.

3. D. T. Absalam-Gadzhievich, B. A. Ramazanovich, Research of Thermal Stability of Water Mixtures of Aliphatic Alcohols. Journal of Materials Science and Engineering A 2 (12) 786790, (2012).

4. L. H. Horsley, Azeotropic Data-III, Washington, D.C: American Chemical Society, ISBN 9780841205338, (1973).

5. ScienceLab. Material Safety Data Sheet Listing. [cited 18.11.13]; Available from: www.sciencelab.com/msdsList.php. 